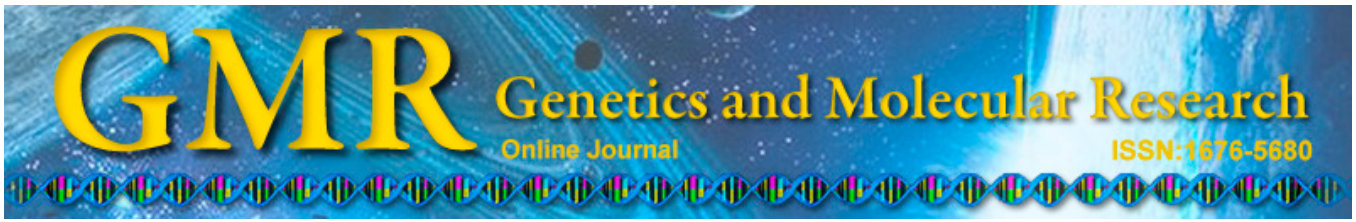

\title{
Estimating additive and dominance variances for complex traits in pigs combining genomic and pedigree information
}

\author{
E.V. Costa ${ }^{1}$, D.B. Diniz ${ }^{1}$, R. Veroneze ${ }^{1}$, M.D.V. Resende ${ }^{2}$, C.F. Azevedo ${ }^{3}$, \\ S.E.F. Guimaraes ${ }^{1}$, F.F. Silva ${ }^{1}$ and P.S. Lopes ${ }^{1}$ \\ ${ }^{1}$ Departamento de Zootecnia, Universidade Federal de Viçosa, Viçosa, \\ MG, Brasil \\ ${ }^{2}$ EMBRAPA Florestas, Colombo, PR, Brasil \\ ${ }^{3}$ Departamento de Estatística, Universidade Federal de Viçosa, Viçosa, \\ MG, Brasil \\ Corresponding author: P.S. Lopes \\ E-mail: plopes@ufv.br
}

Genet. Mol. Res. 14 (2): 6303-6311 (2015)

Received July 31, 2014

Accepted November 17, 2014

Published June 11, 2015

DOI http://dx.doi.org/10.4238/2015.June.11.4

\begin{abstract}
Knowledge of dominance effects should improve genetic evaluations, provide the accurate selection of purebred animals, and enable better breeding strategies, including the exploitation of heterosis in crossbreeds. In this study, we combined genomic and pedigree data to study the relative importance of additive and dominance genetic variation in growth and carcass traits in an F2 pig population. Two GBLUP models were used, a model without a polygenic effect (ADM) and a model with a polygenic effect (ADMP). Additive effects played a greater role in the control of growth and carcass traits than did dominance effects. However, dominance effects were important for all traits, particularly in backfat thickness. The narrow-sense and broadsense heritability estimates for growth $(0.06$ to 0.42 , and 0.10 to 0.51 , respectively) and carcass traits ( 0.07 to 0.37 , and 0.10 to 0.76 , respectively) exhibited a wide variation. The inclusion of a polygenic effect in the ADMP model changed the broad-sense heritability estimates only
\end{abstract}


for birth weight and weight at 21 days of age.

Key words: Dominance; Genetic Parameters; Polygenic effect; Piau pigs

\section{INTRODUCTION}

Complex traits are controlled by many genes, with additive and non-additive effects, and by environmental factors. The additive genetic expression of these traits is the result of the cumulative effect of numerous genes and non-additive genetic variation, including dominance and epistasis, resulting from interactions between alleles within and between loci, with the dominance effect being the most important (Gengler et al., 1998).

The partition of phenotypic variance into dominance and additive genetic effects removes some of the confounding factors, such as a common maternal environment, that would otherwise bias the results of an analysis (Mrode and Thomson, 2005). Therefore, the estimation of dominance variance allows an unbiased estimation of heritability in the narrow sense, and a more precise prediction of additive effects and the use of dominance effects through a crossbreeding or special mating strategy (Wei and van der Werf, 1993).

Traditionally, the estimation of dominance variance in livestock populations has focused on phenotypic and pedigree data (Culbertson et al., 1998; Ishida et al., 2001; Serenius and Stalder, 2006; Angkuraseranee, 2010). The estimation of these effects using genomic data is usually neglected, despite its potential to obtain more accurate and reliable estimates of variance components compared to pedigree-based analysis (Lee et al., 2010). Su et al. (2012) estimated the non-additive genetic variance in daily gain in Danish Duroc purebred pigs, using a genomic-based approach. However, dominance variation in crossbreeds is expected to be much larger; therefore, the fraction of phenotypic variance attributed to dominance effects in crossbreeds at the genomic level remains unknown. In addition, Su et al. (2012) only evaluated one trait (daily gain); however, the effects of dominance depend on the trait studied, so it is important to evaluate dominance effects in a range of traits relevant to the pig industry.

Knowledge of dominance effects should improve genetic evaluations, provide the accurate selection of purebred animals, and enable better breeding strategies, including the exploitation of heterosis in crossbreeds. Therefore, the fraction of the phenotypic variance that can be attributed to dominance effects must be separated from the additive effects fraction. In the present study, we combined genomic and pedigree data to study the relative importance of additive and dominance genetic variation in growth and carcass traits in a crossbred pig population.

\section{MATERIAL AND METHODS}

All procedures with animals were carried out in accordance with the Ethics Statements of the Departamento de Zootecnia, Universidade Federal de Viçosa (UFV), MG, Brazil.

The experiment was conducted at the pig genetic breeding farm at Universidade Federal de Viçosa, Viçosa, MG, Brazil. The F1 population was generated by crossing two boars of the local Piau pig breed with 18 commercial sows (Landrace $\mathrm{x}$ Large White $\mathrm{x}$ Pietrain). A random selection of $11 \mathrm{~F} 1$ boars and 54 F1 dams was conducted. These animals were crossed to create an F2 population. The phenotypic data consisted of eight carcass and seven growth traits taken from the F2 animals. Table 1 presents the means and standard deviations of all the traits. 
Table 1. Means, standard deviations (SD), and coefficients of variation (CV) for carcass and growth traits.

\begin{tabular}{lcccc}
\hline Trait & $\mathrm{N}$ & Means & SD & CV (\%) \\
\hline CW $(\mathrm{kg})$ & 414 & 53.33 & 5.38 & 10.09 \\
RHCW $(\mathrm{kg})$ & 416 & 26.68 & 2.83 & 4.78 \\
MLC $(\mathrm{cm})$ & 414 & 71.59 & 3.42 & 13.67 \\
SBT $(\mathrm{mm})$ & 416 & 40.56 & 5.54 & 24.36 \\
LR $(\mathrm{mm})$ & 417 & 19.52 & 4.75 & 25.89 \\
LL $(\mathrm{mm})$ & 415 & 23.10 & 5.98 & 15.15 \\
P2 $(\mathrm{mm})$ & 416 & 16.83 & 3.75 & 22.50 \\
LEA $(\mathrm{cm})$ & 381 & 26.40 & 4.00 & 21.71 \\
BW $(\mathrm{kg})$ & 333 & 1.20 & 0.27 & 22.24 \\
W21 $(\mathrm{kg})$ & 399 & 4.91 & 1.07 & 21.08 \\
W42 $(\mathrm{kg})$ & 400 & 8.31 & 1.85 & 20.44 \\
W63 $(\mathrm{kg})$ & 410 & 16.23 & 3.42 & 18.38 \\
W77 $(\mathrm{kg})$ & 414 & 21.35 & 4.37 & 9.22 \\
W105 $(\mathrm{kg})$ & 408 & 64.14 & 6.64 & 5.97 \\
SW $(\mathrm{kg})$ & 406 & 64.79 & & \\
\hline
\end{tabular}

The evaluated traits were: CW, hot carcass weight, including feet and head; RHCW, right carcass weight; MLC, carcass length, measured using the American carcass classification method; SBT, higher backfat thickness in the shoulder region; LR, midline backfat thickness, immediately after the last rib; LL, backfat thickness at the last lumbar vertebra; P2, backfat thickness at the last rib, $6.5 \mathrm{~cm}$ from the midline; LEA, loin eye area; BW, birth weight; W21, weight at 21 days of age; W42, weight at 42 days of age; W63, weight at 63 days of age; W77, weight at 77 days of age; W105, weight at 105 days of age; SW, slaughter weight.

DNA was extracted from white blood cells of grandparental, F1, and F2 animals at the Laboratório de Biotecnologia Animal, Departamento de Zootecnia, Universidade Federal de Viçosa. A detailed description of the procedures can be found in Band et al. (2005). From the F2 population, 345 animals were genotyped for 384 single nucleotide polymorphisms (SNPs). The low-density SNP chips were customized selecting markers (Illumina PorcineSNP60 BeadChip, San Diego, CA, USA; Ramos et al., 2009). These markers spanned the regions where quantitative trait loci (QTLs) had previously been detected in this population (Hidalgo et al., 2013). The SNPs were submitted to a quality control wherein 66 markers were discarded due to non-amplification and 81 were discarded due to a low Minor Allele Frequency (MAF < 0.05). A total of 237 SNPs remained, which were distributed as follows: SSC1 (56), SSC4 (54), SSC7 (59), SSC8 (31), SSC17 (25), and SSCX (12). Two GBLUP models were used to compute genetic parameters - a model without inclusion of the polygenic effect (ADM), and a model with inclusion of the polygenic effect (ADMP):

$$
\begin{gathered}
y=X b+Z a+W d+e(A D M) \\
y=X b+Z a+W d+T g+e(A D M P)
\end{gathered}
$$

(Equation 1)

(Equation 2)

where $y$ is the vector of observations; $b$ is the vector of non-genetic effects, which include the effects of sex, batch, and halothane genotype; $a$ is the vector of additive genetic effects (breeding values); $d$ is the vector of dominance deviation effects; $g$ is the vector of polygenic infinitesimal effects; and e is the vector of random residuals. $X, Z, W$, and $T$ are incidence matrices. It is assumed that

$$
\begin{aligned}
& \mathrm{a} \sim \mathrm{N}\left(0, \mathrm{G} \sigma_{\mathrm{a}}^{2}\right) \\
& \mathrm{d} \sim \mathrm{N}\left(0, \mathrm{G}_{\mathrm{d}} \sigma_{\mathrm{d}}^{2}\right) \\
& g \sim \mathrm{N}\left(0, A \sigma_{\mathrm{g}}^{2}\right)
\end{aligned}
$$

(Equation 5) 


$$
e \sim \mathrm{N}\left(0, \mathrm{I} \sigma_{\mathrm{e}}^{2}\right)
$$

(Equation 6)

$G$ is the genomic-based additive relationship matrix, and can be constructed using SNP marker information according to VanRaden (2008),

$$
G=\frac{M M \prime}{\sum 2 p i q i}
$$

where $M$ is an $n \times$ m matrix ( $n=$ number of animals, $m=$ number of marker loci) that specifies the SNP genotype at each locus for each animal. $G_{d}$ is the genomic-based dominance genetic matrix,

$$
G_{d}=\frac{S S}{\sum\left(2 p_{i} q_{i}\right)^{2}}
$$

where an S matrix is composed of the values $\left(-2 p^{2}\right),(2 p q)$, and $\left(-2 q^{2}\right)$ to the genotypes of marker types mm, Mm, and MM, respectively (Vitezica et al., 2013).

A Bayesian additive-dominance G-BLUP, or the Bayesian regression method, was fitted using the GS3 software (Legarra et al., 2011) via MCMC-REML/BLUP, with flat (-2 degrees of freedom, which turned the inverted chi-square distribution into a uniform distribution for variance components) priors for genetic and environmental variances. This was expected to produce results similar to those of the G-BLUP and RR-BLUP.

We used 100,000 iterations for the MCMC algorithms of the different models, with the first 20,000 iterations discarded as burn-in. After performing each set of 10 iterations (thin), a sample was retained in order to calculate $a$ posteriori statistics. Consequently, 10,000 MCMC samples were used for building a posteriori densities. The convergence of the Markov chains was checked by visualizing the sampling paths, and by running repeated progressive analyses until convergence occurred. The variance of dominance was calculated according to the following equation (Habier et al., 2007):

$$
\sigma_{d}^{2}=\sum_{i=1}^{n}\left(2 p_{i} q_{i}\right)^{2} \sigma_{\tilde{d}}^{2}
$$

(Equation 9)

where $p_{i}$ and $q_{i}$ are the allelic frequencies at the locus $i$ and $\sigma_{\delta i}^{2}$ is the variance of dominance attributed to locus i. The additive variance was computed as follows (Habier et al., 2007):

$$
\sigma_{a}^{2}=\sum_{i=1}^{n}\left(2 p_{i} q_{i}\right) \sigma_{o i}^{2}
$$

where $\sigma_{\alpha i}^{2}$ is the additive variance attributed to locus i.

\section{RESULTS}

The ADM model, which included additive and dominance effects, was used to compute genetic parameters. The estimates of the variance components and heritability for 15 carcass and growth traits are shown in Table 2. 


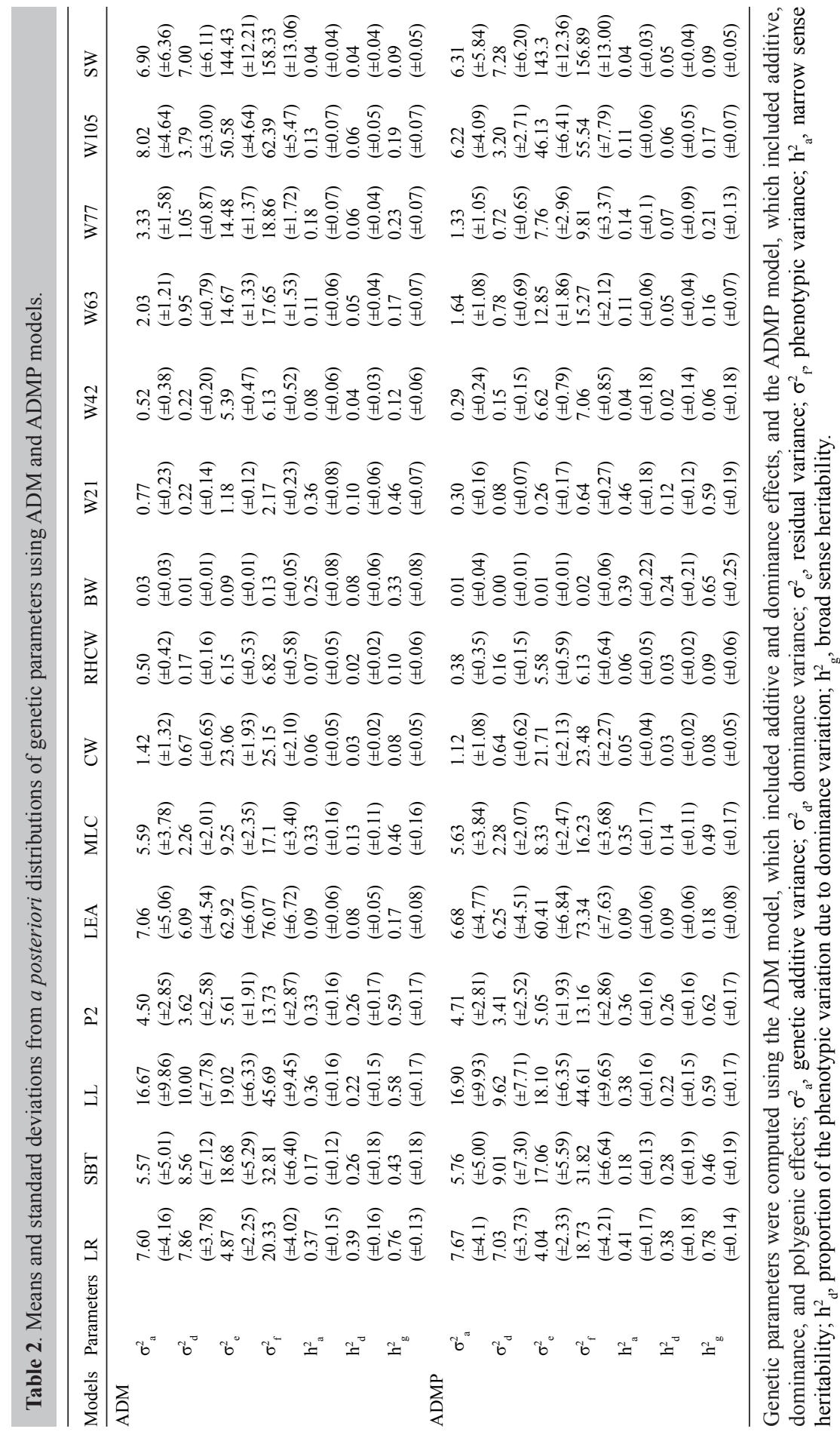


For the dominance effects, the carcass trait LR (midline backfat thickness) exhibited the strongest magnitude, accounting for $37.5 \%$ of the phenotypic variance observed, whereas RHCW (right carcass weight) exhibited the lowest dominance proportion (2.7\%). Concerning additive effects, LR exhibited the strongest narrow-sense heritability (0.37), whereas CW (hot carcass weight) had the lowest estimate (0.07). For the broad-sense heritability, LR had the highest estimate (0.76), whereas $\mathrm{CW}$ had the lowest $(0.10)$.

For the growth traits, W21 (weight at 21 days of age) exhibited the strongest dominance effect, accounting for $10 \%$ of the phenotypic variance observed, while W42 (weight at 42 days of age) had the lowest effect (4\%). Regarding the narrow and broad-sense heritabilities, W21 had the highest estimates ( 0.42 and 0.51 , respectively), and SW (slaughter weight) had the lowest estimates ( 0.06 and 0.10 , respectively).

Dominance effects were more pronounced for the following carcass traits: LR, SBT (backfat thickness in the shoulder region), LL (backfat thickness at the last lumbar vertebra), and P2 (backfat thickness at the last rib, $6.5 \mathrm{~cm}$ from the midline) (Figure 1A). Dominance effects were expressed less than additive effects in all of the growth traits analyzed (Figure 1B).

According to Solberg et al. (2009), when a sparse marker map is used the inclusion of a polygenic effect can capture genetic variation that is not in tight linkage with markers. To test if the inclusion of the polygenic effect in the model modified the estimates of the genetic parameters, the ADMP model with this effect was tested. The estimates of variance components and heritability resulting from the inclusion of the polygenic effect for the same 15 carcass and growth traits are shown in Table 2. The narrow and broad-sense heritabilities, and ${ }_{h_{d}^{2}}$, for the carcass traits exhibited the same trend in both models. However, for the growth traits BW (birth weight) and W21, the narrow and broad-sense heritabilities and $h_{d}^{2}$ exhibited different trends in the two models: the narrow-sense heritability and $h_{d}^{2}$ results for these traits obtained from the ADM model were lower than those obtained from the ADMP model.

\section{DISCUSSION}

In this study, we partitioned genotypic variance between additive and dominance effects in complex traits, using genomic and pedigree information of a crossbreed pig population. In general, additive effects played a greater role in the control of growth and carcass traits than did dominance effects. Except for BW and W21, the narrow-sense heritabilities of all of the traits were similar to those previously reported (Ishida et al., 2001).

In relation to the total variance, dominance effects were important in backfat thickness, LR, SBT, LL, and P2. The more pronounced dominance effects observed for the carcass traits can be explained by the use of the local Brazilian Piau pig breed, which exhibits a high fat deposition, to generate the F2 population. This breed has never undergone a breeding and selection process for growth and carcass traits, and is completely divergent from commercial lines. Dominance effects should be more pronounced in crossbreed populations (Orengo et al., 2009), and the more divergent the parental population, the higher the expected magnitude of this effect, due to the increase in heterozygosity in the crossbred population.

We also found a wide variation in dominance levels for the carcass traits in the crossbreed population analyzed. Using pedigree data from purebreed Landrace pigs, Ishida et al. (2001) reported that the proportion of dominance variance to total genetic variance for carcass traits ranged from 0 to 0.86 . Working with simulated data, Wittenburg et al. (2011) concluded 
that the estimation of additive and non-additive effects (particularly dominance) affects the accuracy of predictions, and the proportion of genetic variation attributed to additive effects. Therefore, although dominance effects did not affect growth traits in our study, the inclusion of dominance in our variance estimation model could have improved the accuracy of predicting additive effects.
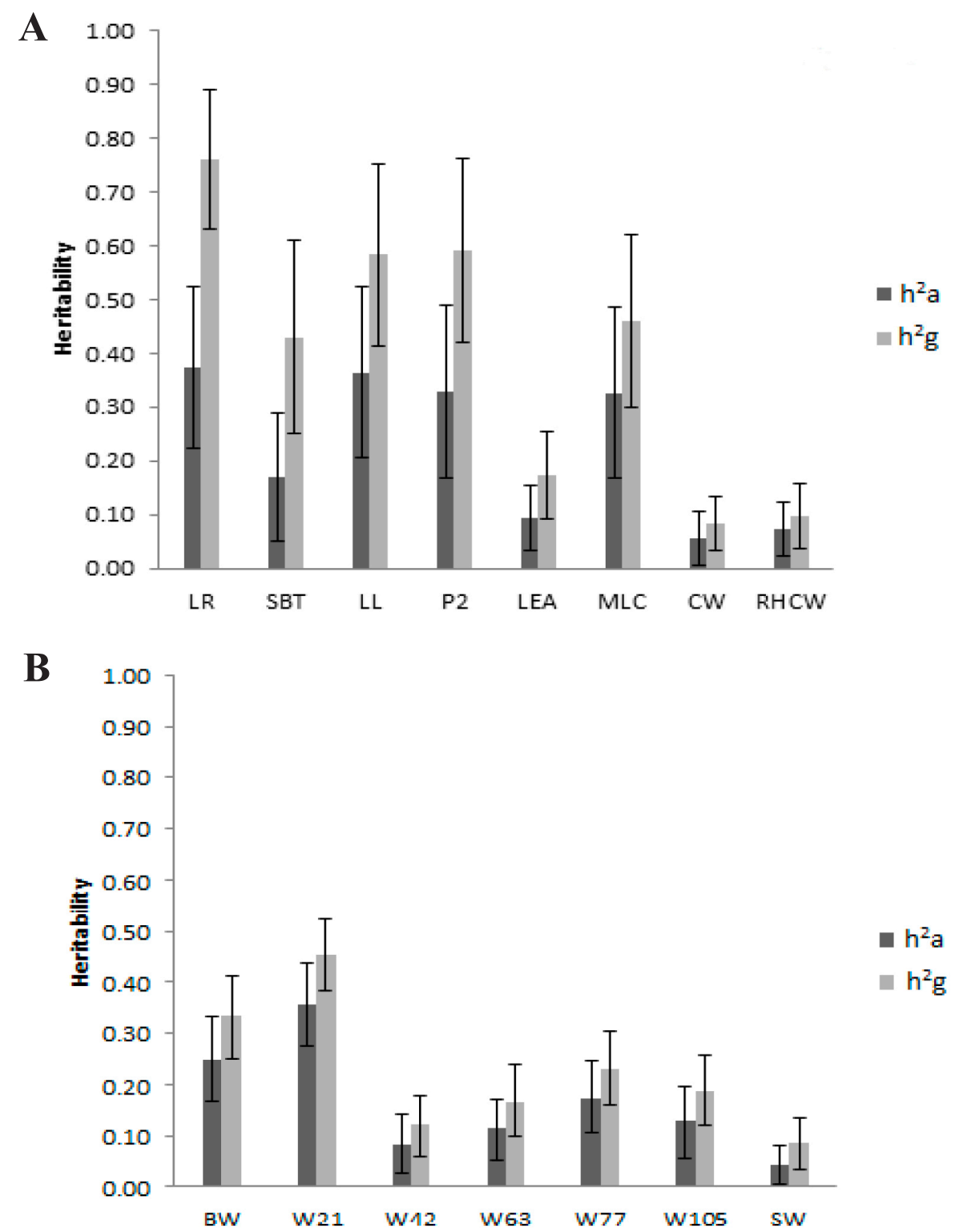

Figure 1. Means and standard deviations from a posteriori distributions of broad $\left(h_{g}^{2}\right)$ and narrow $\left(h_{a}^{2}\right)$ sense heritabilities for carcass (A) and growth (B) traits. 
The narrow and broad-sense heritabilities varied widely between the carcass and growth traits. For the carcass traits LR, SBT, LL, and P2, we found a considerable difference in the magnitude of broad-sense heritability compared to narrow-sense heritability. By contrast, we did not find any great differences in the magnitudes of the heritabilities for the growth traits.

Hidalgo et al. (2013), working with the same population of pigs as used in our study, detected QTLs with dominance effects for the carcass traits MLC (carcass length, measured using the American carcass classification method), LEA (loin eye area), LR, and SBT, and for the growth traits BW, W63 (weight at 63 days of age), and W77 (weight at 77 days of age), in chromosomes $1,4,7,8$, and 17 . Our results corroborate these findings, demonstrating the occurrence of dominance effects for these traits. Also working with simulated data, Calus and Veerkamp (2007) concluded that the inclusion of a polygenic effect, in addition to marker information in variance estimation models, removes the bias from the estimated variance components. In the present study, the inclusion of a polygenic effect in the ADMP model changed the broad-sense heritabilities for the growth traits BW and W21. This may have been because the polygenic effect captured the variance that was not explained by the markers for these traits. For the other growth and carcass traits studied, the marker panel that was used acceptably explained the total variance. The observed lack of change in the polygenic effect trend may be because these markers were located in regions where QTLs have been found in previous studies in this population (Paixão et al., 2008; Silva et al., 2010; Sousa et al., 2011; Hidalgo et al., 2013). The markers captured the majority of the genes controlling such traits, as a result of linkage disequilibrium with them.

A limitation of the approach taken was the small number and sparse distribution of SNP markers throughout the genome. However, as mentioned above, the markers were distributed in QTL regions, creating an SNP marker panel that was able to capture the genetic variance of the traits in this population. Despite the relatively small number of animals evaluated, the population was structured with an F2 design, which results in larger linkage disequilibrium blocks that improve the capture of genetic variance, even in low-density marker panels.

In conclusion, this study revealed that the partition of genetic variance into additive and dominance components improves our knowledge of the genetic control of important carcass and growth traits in crossbred pigs. The dominance effect was important in all of the traits studied, particularly in backfat thickness. This is the first study that has estimated dominance variance in a large number of carcass and growth traits.

Knowledge of the genetic effects that control economically important traits in pigs is essential to improve the efficiency of the production system. Therefore, the data reported here may be relevant for the development of more accurate models for the prediction of animal breeding values, and crossbreeding performance. In future studies, these findings can be tested in other crossbred populations, including different breeds genotyped with a high-density SNP panel, with the inclusion of epistatic effects in the model.

\section{ACKNOWLEDGMENTS}

Research supported by Coordenação de Aperfeiçoamento de Pessoal de Nível Superior (CAPES), Conselho Nacional de Desenvolvimento Científico e Tecnológico (CNPq), and Fundação de Amparo à Pesquisa do Estado de Minas Gerais (FAPEMIG). 


\section{REFERENCES}

Angkuraseranee T (2010). Estimation of additive and dominance variance for reproductive traits from different models in Duroc purebred. Songklanakarin J. Sci. Technol. 32: 115-117.

Band GDO, Guimarães SEF, Lopes PS, Peixoto JDO, et al. (2005). Relationship between the Porcine Stress Syndrome gene and carcass and performance traits in F2 pigs resulting from divergent crosses. Genet. Mol. Biol. 28: 92-96.

Calus MPL and Veerkamp RF (2007). Accuracy of breeding values when using and ignoring the polygenic effect in genomic breeding value estimation with a marker density of one SNP per cM. J. Anim. Breed. Genet. 124: 362-368.

Culbertson M, Mabry JW, Misztal I, Gengler N, et al. (1998). Estimation of dominance variance in purebred Yorkshire swine. J. Anim. Sci. 76: 448-451.

Gengler N, Misztal I, Bertrand J and Culbertson M (1998). Estimation of the dominance variance for postweaning gain in the US Limousin population. J. Anim. Sci. 76: 2515-2520.

Habier D, Fernando RL and Dekkers JCM (2007). The impact of genetic relationship information on genome-assisted breeding values. Genetics 177: 2389-2397.

Hidalgo AM, Lopes PS, Paixão DM, Silva FF, et al. (2013). Fine mapping and single nucleotide polymorphism effects estimation on pig chromosomes 1, 4, 7, 8, 17 and X. Genet. Mol. Biol. 36: 511-519.

Ishida T, Kuroki T, Harada H and Fukuhara R (2001). Estimation of additive and dominance genetic variances in line breeding swine. Asian-Australasian J. Anim. Sci. 14: 1-6.

Lee SH, Goddard ME, Visscher PM and van der Werf JH (2010). Using the realized relationship matrix to disentangle confounding factors for the estimation of genetic variance components of complex traits. Genet. Sel. Evol. 42: 22.

Legarra A, Ricardi A and Filangi O (2011). GS3: Genomic Selection, Gibbs Sampling, Gauss-Seidel (and BayesCp).

Mrode R and Thomson R (2005). Linear models for the prediction of animal breeding values II. Cab International, MA, USA.

Orengo J, Piles M, Rafel O, Ramon J, et al. (2009). Crossbreeding parameters for growth and feed consumption traits from a five diallel mating scheme in rabbits. J. Anim. Sci. 87: 1896-1905.

Paixão DM, Silva Filho MID, Pereira MS, Lopes MS, et al. (2008). Quantitative trait loci for carcass, internal organ and meat quality traits on porcine chromosomes 16,17 and 18. Genet. Mol. Biol. 31: 898-901.

Ramos AM, Crooijmans RPMA, Affara NA, Amaral AJ, et al. (2009). Design of a high density SNP genotyping assay in the pig using SNPs identified and characterized by next generation sequencing technology. PLoS One. 4: e6524.

Serenius T and Stalder K (2006). Selection for sow longevity. J. Anim. Sci. 84: 166-171.

Silva KM, Bastiaansen JWM, Knol EF, Merks JWM, et al. (2010). Meta-analysis of results from quantitative trait loci mapping studies on pig chromosome 4. Anim. Genet. 42: 280-292.

Solberg TR, Sonesson AK, Woolliams JA, Odegard J, et al. (2009). Persistence of accuracy of genome-wide breeding values over generations when including a polygenic effect. Genet. Sel. Evol. 41: 53.

Sousa KRS, Guimarães SEF, Filho MIS, Lopes MS, et al. (2011). Mapeamento de locos de características quantitativas nos cromossomos 5, 7 e 8 de suínos. Rev. Bras. Zootec. 40: 115-123.

$\mathrm{Su}$ G, Christensen OF, Ostersen T, Henryon M, et al. (2012). Estimating additive and non-additive genetic variances and predicting genetic merits using genome-wide dense single nucleotide polymorphism markers. PloS One 7: e45293.

Vitezica ZG, Varona L and Legarra A (2013). On the additive and dominant variance and covariance of individuals within the genomic selection scope. Genetics 195: 1223-1230.

Wei M and van der Werf JH (1993). Animal model estimation of additive and dominance variances in egg production traits of poultry. J. Anim. Sci. 71: 57-65.

Wittenburg D, Melzer N and Reinsch N (2011). Including non-additive genetic effects in Bayesian methods for the prediction of genetic values based on genome-wide markers. BMC Genet. 12: 74. 